\title{
EXPERIMENTAL INVESTIGATION OF SPRAY COMBUSTION REGIMES IN AEROENGINE COMBUSTORS
}

\author{
M. Vicentini ${ }^{1}$, R. Lecourt ${ }^{1}$, O. Rouzaud ${ }^{1}$, V. Bodoc ${ }^{1}$, \\ and O. Simonin ${ }^{2}$ \\ ${ }^{1}$ ONERA \\ 2 Av. Edouard Belin, Toulouse Cedex 31000, France \\ ${ }^{2} \mathrm{IMFT}$ \\ Allée du Professeur Camille Soula, Toulouse Cedex 31400, France
}

\begin{abstract}
At ONERA Fauga-Mauzac center, a new air-breathing propulsion test setup, Prométhée-LACOM, has been recently developed. In this paper, both nonreacting and reacting two-phase flows (nonpremixed spray) were investigated. Under reactive conditions, simultaneous OH-PLIF (planar laser-induced fluorescence) and Mie scattering imaging were implemented in order to characterize the spray flame structure. The different behaviors observed in this study seem to support the existence of spray combustion regimes. Moreover, statistical analysis was performed on the spatial distribution of droplets and indicated that the center-to-center interdroplet distance (nearest neighbor) could be described by means of a perfectly random distribution.
\end{abstract}

\section{INTRODUCTION}

Turbulent combustion is widespread in engineering applications such as gas turbines, internal combustion engines, rocket motors and still contributes significantly to our energy supply. Typically in aeronautical propulsion devices, liquid fuels - with high energy density — are used in order to optimize storage volumes and then increase ranges of aircraft. At the inlet of the combustion chamber, the liquid fuel must be atomized into a spray of small droplets to increase the exchange surface area between fuel and hot gases. Actually, the combustion efficiency depends strongly on the quality of fuel pulverization, fuel-oxidant mixing, and chemical kinetics processes. Even though the specific fuel consumption of recent turbofan engines has decreased, pollutant emissions levels remain high and 
are increasingly subject to environmental taxes in the transport sector $(\mathrm{NOx}$, $\mathrm{CO}$, and unburned hydrocarbons). Because of this major issue, investigations of spray combustion are quite numerous.

Chiu and Kim [1] and Chigier [2] developed the pioneering concept of "spray combustion" regimes. According to their theory, within dense zones of a cloud, the droplets vaporize collectively and the front flame stands beyond the outer boundary of the spray. Indeed, the core of those zones is nonflammable because of a rich mixture. In contrast, when the droplet spacing is sufficiently great, then hot gases and oxidant can penetrate into the cloud and potentially ignite single droplets. Thus, in an intermediate number density of droplets, a "group combustion" regime is likely (i.e., diffusion flame enclosing cluster of droplets). Most of available works are aimed at theoretical and numerical analysis [3-6]. Some experimental investigations on this topic also exist. Akamatsu and coworkers $[7,8]$ studied a premixed spray flame stabilized by an annular hydrogen flame pilot. They implemented simultaneous local measurements such as Phase Doppler Anemometer (PDA) and photomultipliers (Mie scattering and light emission of $\mathrm{OH}$ - and $\mathrm{CH}$-radicals). The authors found that the uniformly premixed spray stream was fragmented into droplet clusters due to preferential through easy-to-burn routes in the reacting flow. More recently, Beck and his colleagues $[9,10]$ studied a partially prevaporized swirlstabilized spray flame. They used CH-PLIF and Mie scattering visualizations in order to capture single droplet burning modes. Besides, 3D PDA permitted measuring the slip velocities of droplets. Two modes were identified: envelope flame and wake flame, depending on the slip velocity. Finally, the authors suggested that wake flames produce less NOx emissions than envelope flames due to an increase of the mixing length between the surface of the droplet and its flame.

In this article, the focus is on the experimental study of these spray combustion regimes. In this purpose, a new air-breathing propulsion test setup has been developed. In the next section, the test setup design and the implementation of measurements are presented. An important point concerns the setting of simultaneous optical techniques to obtain superimposed visualizations of both droplets and reacting zones. Section 3 presents the results of both nonreacting and reacting flows. The aim is to build an experimental database to improve combustion modeling in numerical codes. Moreover, the present authors underline combustion effects on the flow behavior (i. e., gaseous phase instabilities, droplet sizes, and velocities) compared to the nonreacting flow. A specific analysis on the spatial distribution of droplets under reactive conditions is also carried out. By doing so, spray heterogeneities are highlighted and droplet spacing distributions are described by means of a probability density function (pdf). Furthermore, experimental evidences supporting the existence of spray combustion regimes are provided. It seems that the interdroplet distances play a major role in the appearance of those behaviors. 


\section{MATERIALS AND METHODS}

\section{$2.1 \quad$ Test Setup}

\subsubsection{An overview}

At ONERA Fauga-Mauzac center, a new air-breathing propulsion test setup, Prométhée-LACOM, has been recently developed (Fig. 1a). The air feeding system is made up of a spherical pressure vessel (25 bar), gas pressure regulators, and an electric heater $(1 \mathrm{MW})$. The air mass flow rate is measured with a sonic nozzle located downstream of the electric heater $( \pm 3.3$ percent accuracy). Subsequently, the preheated air stream passes through a succession of flow conditioners and grids for generating turbulence. The test chamber consists of a square internal section $(120 \times 120 \mathrm{~mm})$. As it can be seen from Fig. $1 b$, the confined turbulent air stream flows around a fuel injection system prior to the combustion chamber. The injection system consists in a bluff-body in which a liquid fuel nozzle is mounted. It should be noted that the bluff-body spans over

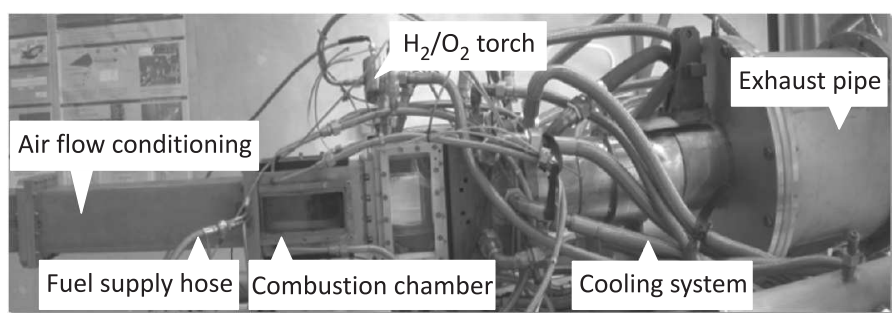

(a)

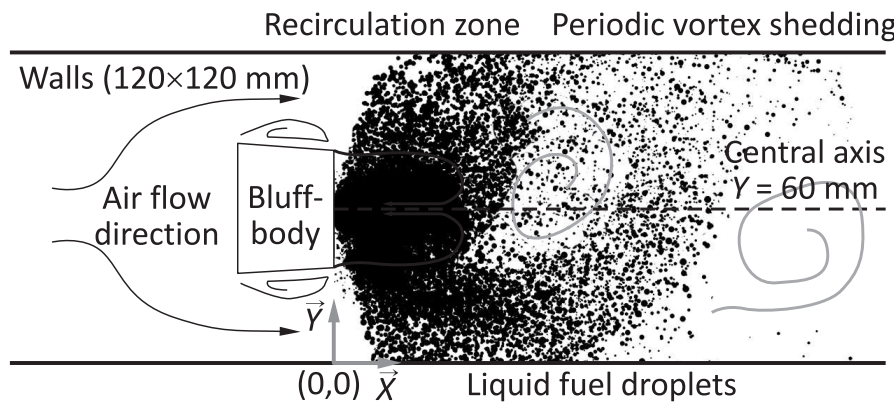

(b)

Figure 1 Prométhée-LACOM test setup: (a) the facility; and (b) illustration of the nonreacting two-phase flow 
the entire channel. The fuel feeding line $(1.5 \mathrm{MPa})$ is equipped with a Coriolis flowmeter ( \pm 0.2 percent accuracy). The spray nozzle is centered and screwed into the rear face of the bluff-body. As illustrated in Fig. 1b, a spray of droplets is generated downstream directly into the combustor. The fuel injector consists in a flat fan nozzle which produces an elliptical-shaped spray in the vertical midplane of the combustor, in the $X$-direction. The injection orifice is located at $X=0$ and $Y=60 \mathrm{~mm}$. In addition, the opening angles of the major and minor axes are $150^{\circ}$ and $25^{\circ}$, respectively. Note that the wide angle is set up so as to spray towards the upper and lower walls of the combustor. The water-cooled combustor is equipped with three interchangeable ultraviolet (UV) transparent windows allowing to perform optical measurements downstream of the injection system. Ignition of the air-fuel mixture is triggered by a hydrogen-oxygen torch. Finally, the burnt gases are ejected in the exhaust pipe.

\subsubsection{Specific features}

A previous sizing study has been performed on a prototype in 1 : 1 scale to choose the bluff-body geometry [11]. For a nonreacting flow, this study highlights establishment of a quasi-periodic generation of large-scale eddies with opposite sense of rotation, so-called a von Kármán vortex sheet. As a result, a trapezoidal bluff-body (42 percent ratio blockage, 50-millimeter height, 37-millimeter length, and sharp 4 degree edges) is chosen as optimal geometry in terms of constancy of Strouhal number and signal intensity. For a $5.8 \mathrm{~m} / \mathrm{s}$ bulk velocity, the particle image velocimetry (PIV) measurements reveal a main recirculation zone in the bluff-body's wake, as illustrated in Fig. $1 b$. The average recirculation length is about $45 \mathrm{~mm}$.

Because of its bidimensional design, the experimental setup generates a turbulent flow with two-dimensional mean properties. Moreover, the flat spray of droplets is mainly located around the vertical mid-plane of the combustor. So, as the experimental diagnostics are either punctual or planar, the configuration ensures the measurement of spatially correlated flow data (i. e., droplet positions, sizes, velocities, and flame position). Furthermore, in the ProméthéeLACOM test setup, some features of a flow in an actual aeroengine combustor are preserved. Indeed, the air flow is confined, turbulent, and exhibits instabilities and large-scale structures. Besides, the flame is anchored aerodynamically by creating a main recirculation zone. In this study, $n$-decane is selected as surrogate fuel because it has chemical properties close to the kerosene ones (i. e., density, surface tension, and autoignition temperature). The droplet spray generated is polydisperse (droplets from 5 to $150 \mu \mathrm{m}$ ). Finally, the experiments replicate the operating lean-burn conditions of a turbofan engine, during a flight phase at cruising speed, in terms of fuel-air equivalence ratio. 


\subsection{Operating Conditions}

In this paper, both nonreacting and reacting two-phase flows are investigated. The nominal operating point is set at an air mass flow rate equal to $64 \mathrm{~g} / \mathrm{s}$. At the combustor inlet, the air flow is at standard atmospheric pressure, $450 \mathrm{~K}$, and the turbulence intensity is about $5 \%$ (mean kinetic turbulent energy $\approx 0.1 \mathrm{~m}^{2} / \mathrm{s}^{2}$ ). The corrected mass flow rate corresponds to $1.36[\mathrm{~kg} / \mathrm{s}] \cdot \sqrt{K}\left[\mathrm{bar}^{-1}\right]$. Since the combustion section is $120 \times 120 \mathrm{~mm}$, the bulk velocity is about $5.8 \mathrm{~m} / \mathrm{s}$. The liquid-fuel temperature is measured just upstream from the nozzle and is equal to $330 \mathrm{~K}$. The fuel ( $n$-decane, $\mathrm{C}_{10} \mathrm{H}_{22}$ ) is injected at $1 \mathrm{~g} / \mathrm{s}$ to provide a 44 kilowatt thermal power. Therefore, the fuel-air equivalence ratio is around 0.24. The water cooling of the combustion chamber protects the inner walls $(<650 \mathrm{~K})$ to perform long series of tests (several tens of minutes).

\subsection{Implementation of Measurements}

\subsubsection{Main measurements}

The first challenge of this experimental programme is to build a new database in two-phase flow combustion. Table 1 summarizes the main measurements implemented for the test setup. The PIV measurements which allow to assess the velocity fields of the seeded gaseous phase, are the subject of a previous measurement campaign $[11,12]$. The pressure measurements are used to characterize the instabilities of the gaseous phase in the combustor. The chemiluminescence and PLIF imaging are employed to study the flame fronts in the reacting flow. The first technique uses spontaneous chemical excitation of a flame marker unlike the second technique which produces excitation of the marker by means of a laser. Droplets illuminated by a laser sheet generate light Mie scattering. This signal can be captured by a camera in order to evaluate the positions of droplets from images. Finally, application of a two-component Phase Doppler Interferometry (PDI) technique allows to measure simultaneously the instantaneous droplet

Table 1 Experimental measurement campaign of the Prométhée-LACOM test setup

\begin{tabular}{lll}
\hline Conditions & \multicolumn{1}{c}{ Gaseous phase } & Liquid phase \\
\hline \multirow{2}{*}{ Nonreactive } & \multirow{2}{*}{ PIV } & $\begin{array}{l}\text { PDI } \\
\text { Mie scattering }\end{array}$ \\
\hline \multirow{2}{*}{ Reactive } & Chemiluminescence OH* & PDI \\
& OH-PLIF & Mie scattering \\
\hline
\end{tabular}


velocities (longitudinal, transverse) and their sizes. For more details on spray diagnostics, we recommend the lecture of Bachalo's paper [13].

\subsubsection{Phase-averaged measurements (nonreactive conditions)}

In order to characterize the quasi-periodic dynamic of the nonreacting flow, a phase-averaged analysis of liquid phase measurements is carried out. The pressure fluctuations signal at the level of bluff-body is used as reference signal. Actually, the bluff-body is drilled on its upper and lower faces of static pressure holes (1.7-millimeter diameter). Fluctuations of differential pressure between the upper and lower faces are measured by a diaphragm pressure transducer ( \pm 0.86 -kilopascal sensitivity) and this signal is sampled at $40 \mathrm{kHz}$ in parallel of

the measurements. A postprocessing program links the data (i.e., a posteriori agreement for clock synchronization) and, lastly, a phase-averaging enables to extract the spray dynamic with regards to the coherent motion of the gaseous phase (i.e., vortex shedding phenomenon).

\subsubsection{Simultaneous visualizations of OH-PLIF and Mie scattering (reactive conditions)}

Another aim of this study is to describe the flame structure and to highlight the existence of spray combustion regimes in the reacting two-phase flow. For this purpose, simultaneous tomographic visualizations on the reactive continuous phase and the dispersed phase are conducted with a high spatial resolution. The synchronization system of PLIF from hydroxyl radicals (OH-PLIF) and Mie scattering measurements is shown Fig. $2 a$. Firstly, to excite $\mathrm{OH}$ radicals, a millimetric thin UV laser sheet $(\lambda=282.69 \mathrm{~nm})$ is generated at the outlet of a tunable dye laser Quantel TDL+, pumped by a Nd:YAG laser Quantel YG 980. Secondly, to illuminate droplets, a millimetric thin visible laser sheet is produced from the outlet of a high-frequency Nd:YLF laser Quantronix Darwin $(\lambda=527 \mathrm{~nm})$. The two light sheets are superimposed along the droplet spray axis over a 100-millimeter distance. To acquire the $\mathrm{OH}$ fluorescence images, an intensified CCD camera PI-MAX 2 is focused perpendicularly on the face of the laser sheets through the combustor's lateral window. The camera is equipped with a band-pass filter centered at $311 \pm 5 \mathrm{~nm}$. Likewise, a highspeed camera Phantom V341 is positioned aside to capture the Mie scattering of droplets. The angular separation between both videocameras is $11^{\circ}$. A dewarping procedure enables superimposition of the stack of OH-PLIF and Mie scattering visualizations. A maximal spatial deviation of $57 \mu \mathrm{m}$ is measured between the two series of images. The useful overlapping area of both videocameras is equal to $51.2 \times 45.6 \mathrm{~mm}$ corresponding to a $1804 \times 1606 \mathrm{px}$ corrected resolution $(35.2 \mathrm{px} / \mathrm{mm})$. 


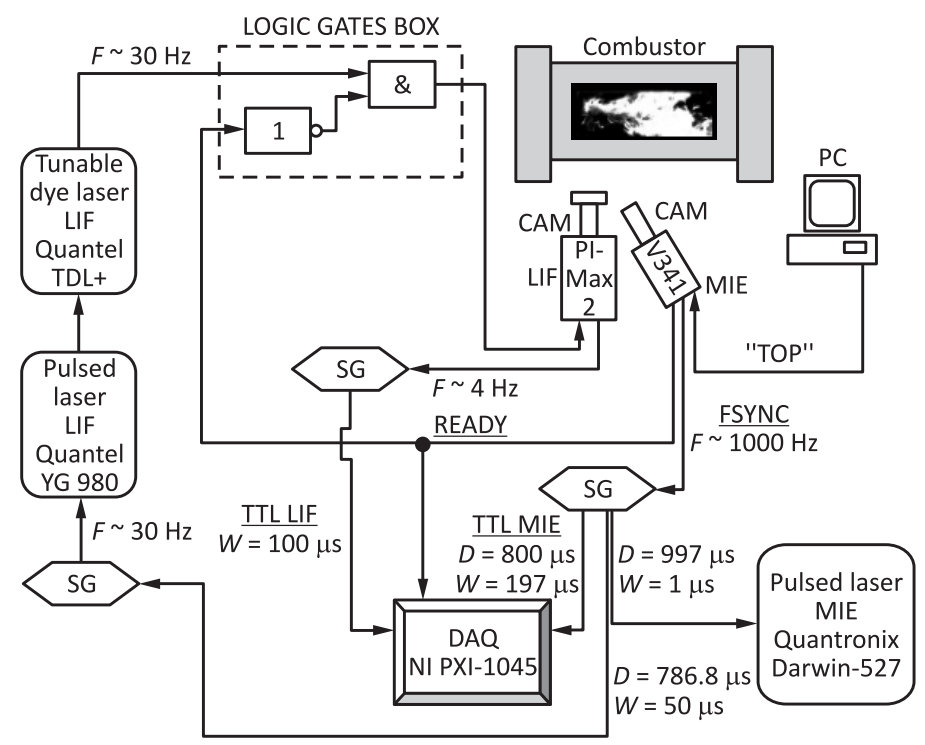

(a)

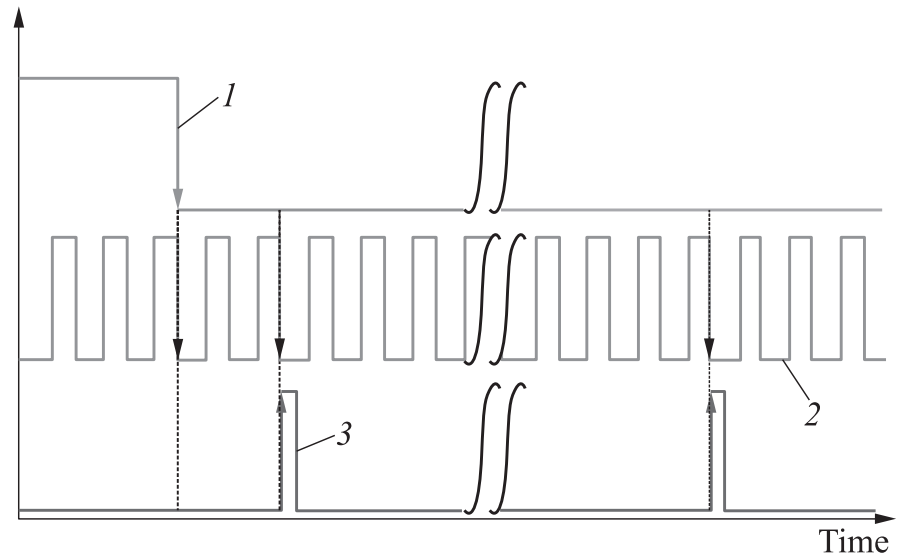

(b)

Figure 2 Setting $(a)$ and digital timing diagram $(b)$ for the synchronization of OH-PLIF and Mie scattering imaging: TTL — transistor-transistor logic; CAM videocamera; SG - signal generator; $D$ - signal's delay; $F$ - signal's frequency; $W$ - signal's width; 1 - Ready signal; 2 - TTL-Mie signal; and 3 - TTL-LIF signal 
A computer is used for triggering the temporal synchronization of both optical techniques. Signal conditioning is applied to electronic camera signals before to be digitized on the data acquisition system (DAQ) $(40 \mathrm{kHz})$. The digital timing diagram is illustrated in Fig. $2 b$. The falling edge of the "Ready" signal corresponds with the beginning of the data recording. From this instant, each falling edge of the "TTL Mie" is associated with a visible laser flash and, consequently, a Mie scattering image (every $1 \mathrm{~ms}$ ). Similarly, each rising edge of the "TTL LIF" signal is associated with an UV flash laser and, thus, with an $\mathrm{OH}-\mathrm{PLIF}$ image (every $250 \mathrm{~ms}$ ). The synchronization system is set to provide a cyclic matching of a falling edge "TTL Mie" with a rising edge "TTL LIF." As a result, a new couple of visualizations is obtained. On the overall network, a maximal 2-microsecond jitter can exist on the image matching time.

\section{RESULTS}

\subsection{Spectral Analysis of the Flow}

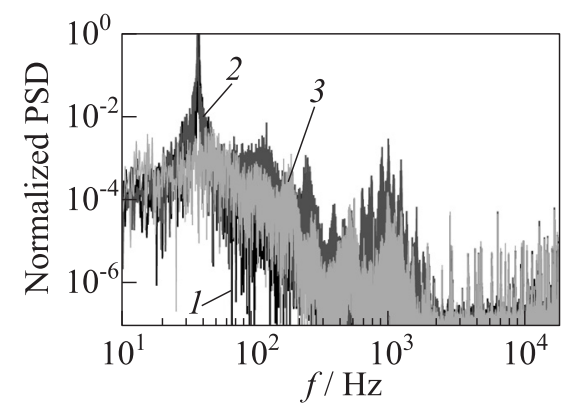

Figure 3 Normalized PSD of the pressure signal: 1 and 2 - nonreacting oneand two-phase flows, respectively; and $3-$ reacting two-phase flow
A spectral analysis of the reference signal (i.e., fluctuations of pressure at the level of the bluff-body) is conducted under the operating conditions. Figure 3 depicts the normalized power spectral densities (PSD) for the one-phase nonreacting flow, the twophase nonreacting flow, and the twophase reacting flow. For the nonreacting case, both spectra (1 and 2) are practically superimposed. One notes that the same narrowband peak frequency at $38 \mathrm{~Hz}$ is highlighted and the Strouhal number based on the height of the bluff-body and the mean flow velocity is equal to 0.32 . Therefore,

the vortex shedding phenomenon seems not perturbed by the liquid droplet loading. In the reacting two-phase flow, nevertheless, the global frequency mode is suppressed (i.e., the peak frequency is no longer observable). This effect has been already disclosed in bluff-body premixed flames [14-16] and can be explained by a decrease of the global vorticity of the flow which is due to the gas expansion and the increase of viscosity effects with temperature. To conclude, under those reactive conditions, heat released by the combustion process is sufficiently high to cause the complete vanishing of the vortex shedding and the flow loses its coherent motion. 

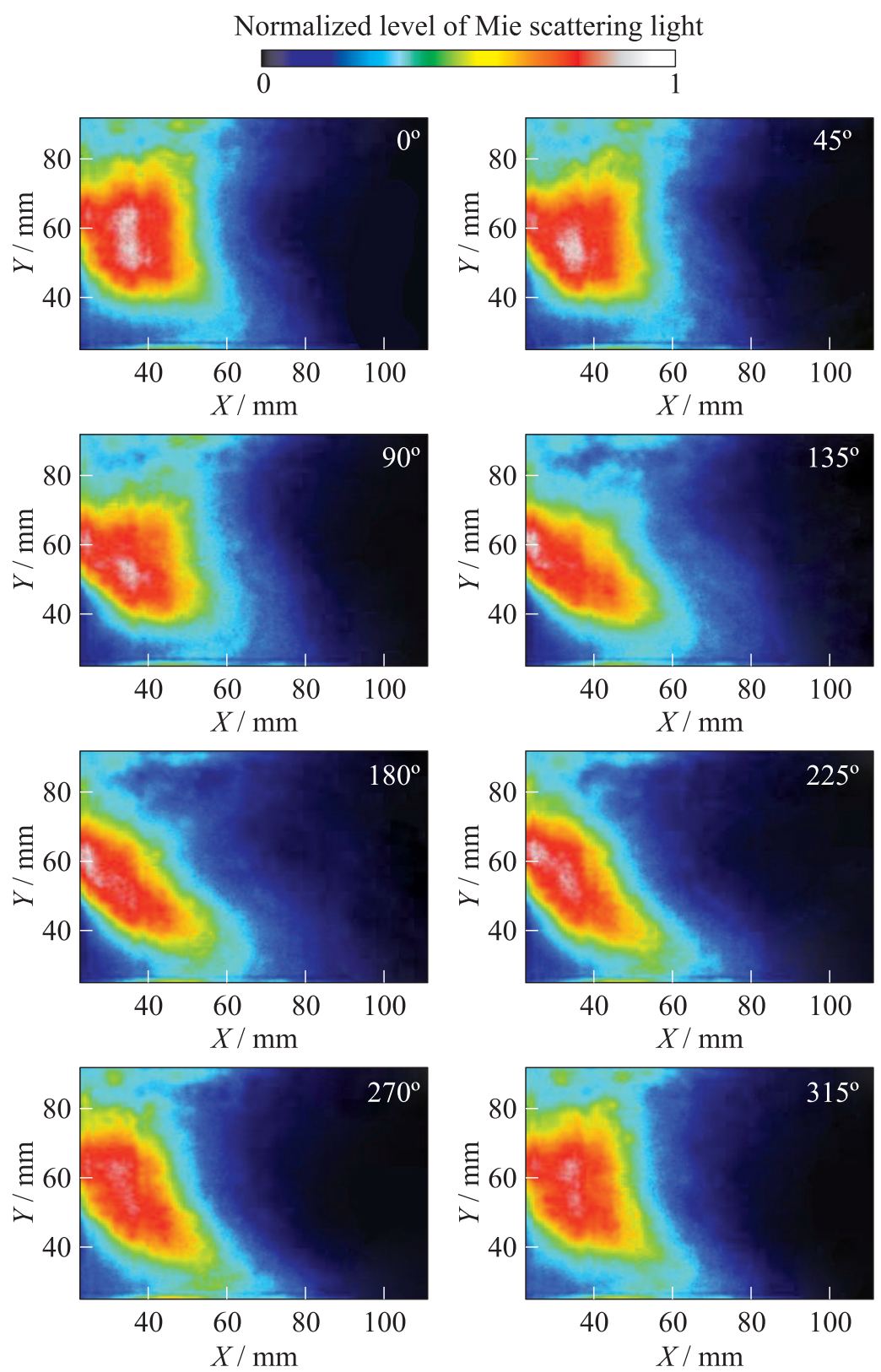

Figure 4 Phase-averaging of Mie scattering images, nonreacting two-phase flow 


\subsection{Spray Visualizations}

To highlight the interaction between the quasi-periodic large-scale structures of the gaseous phase and the spray, under nonreactive conditions, one proceeds phase-averaging of the Mie scattering visualizations. The goal is to "filter" the Brownian motion of the droplets and to extract the unsteady motion of the spray. Figure 4 depicts the data processing, a division into eight phases (every $45^{\circ}$ ) with an accuracy of $4^{\circ}$ was selected. This choice provides 160 instantaneous images per phase. A phase averaging operation consists of averaging pixel by pixel images belonging to the same phase. It is also important to note that a hundred images suffice for converged averages.

The sequence of images shows the spray motion during the vortex shedding cycle, namely, an upward/downward movement in the combustion chamber ( $Y$-direction). One notes also an asymmetry in the intensity of Mie scattering. Indeed, the luminance levels are higher in the bottom of the chamber $(Y<60 \mathrm{~mm})$. This has been explained by a manufacturing defect of the fuel injector which generates a greater quantity of fuel downwards [12].

\subsection{Global Flame Structure}

To determine the global flame structure, the release of $\mathrm{OH}^{*}$ radicals is captured by chemiluminescence. A band-pass filter centered at $311 \pm 5 \mathrm{~nm}$ is used. This filter has a maximal transmission of $50 \%$. In order to offset the light intensity, a Lamber HiCATT image intensifier is mounted between the high-speed camera Phantom V341 $(1 \mathrm{kHz})$ and the system lens plus filter. As shown in Fig. $5 a$,

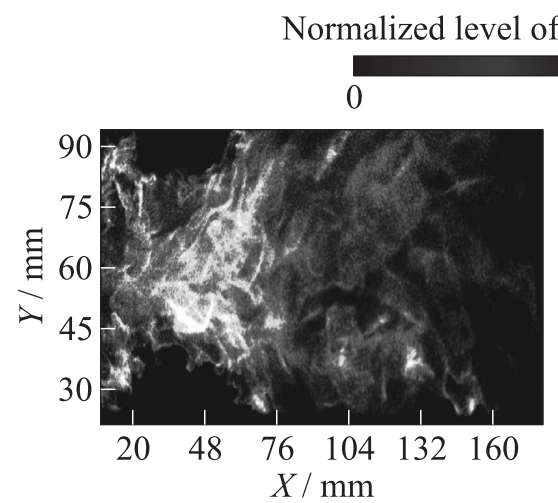

(a)

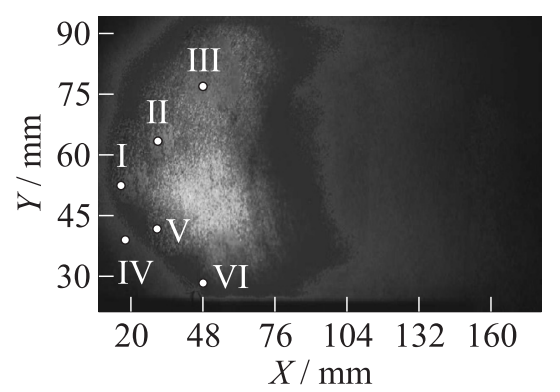

(b)

Figure $5 \mathrm{OH}^{*}$ chemiluminescence instantaneous $(a)$ and time average of $\mathrm{OH}^{*}$ chemiluminescence images $(b)$ 
instantaneous $\mathrm{OH}^{*}$ chemiluminescence visualizations represent the production of hydroxyl radicals integrated along the combustor's depth and reveal the global flame structure. The turbulent nature of the combustion is highlighted by a characteristic flame envelope, i.e., wrinkled, irregular, and unsteady. In these images, one observes significant gradients of $\mathrm{OH}^{*}$ luminescence levels. This behavior is indicative of spray combustion process in which spatial heterogeneities of droplets cause local equivalence ratio fluctuations and intensity disparities of the heat release rate. By applying a fast Fourier transform algorithm on the $\mathrm{OH}^{*}$ luminescence signal, none peak frequency appears in the spectrum. This result reflects the randomness of the reacting flow and corroborates the assumption that von Kármán vortex street has vanished. Averaging the instantaneous visualizations over $4 \mathrm{~s}$ (4000 images), provides a global view of the flame structure presented in Fig. $5 b$. The anchored flame is heart-shaped, or M-shaped, due the recirculation zone of the bluff-body's wake. One can see that chemiluminescence intensity is not symmetric with respect to the central axis of the combustion chamber $(Y=60 \mathrm{~mm})$. Indeed, the heat released in the reacting zone is higher at the bottom of the combustor. Besides, on the average droplet velocity and size profiles, at $9 \mathrm{~mm}$ downstream of the nozzle, this asymmetry can also be observed. Those effects support the assumption of a manufacturing defect of the nozzle as discussed previously.

\subsection{Droplet Size Distributions and Velocities}

Two PDI measurements sections, in the mid-axis of the combustion chamber, are investigated. As a result, the average longitudinal and transverse velocities of droplets and their arithmetic mean diameters are plotted in Fig. 6. Because of the height of the combustor's windows, the sections are limited between $Y$ $=[32 ; 82] \mathrm{mm}$. The spatial measurement discretization is equal to $2 \mathrm{~mm}$ for the nonreactive case and $4 \mathrm{~mm}$ for the reactive case. The first section is located at $X=9 \mathrm{~mm}$ downstream from the fuel nozzle. Under reactive conditions, this section is just upstream the average position of the flame, as seen in Fig. $6 a$. The second section is located at $X=53 \mathrm{~mm}$ downstream the injector and inside the flame region (Fig. $6 b$ ).

On the central axis, at $9 \mathrm{~mm}$ downstream from the injection face, the arithmetic mean diameter $\left(D_{10}\right)$ and the Sauter mean diameter $\left(D_{32}\right)$ are, respectively, 31 and $48 \mu \mathrm{m}$ for the nonreacting flow as opposed to 38 and $51 \mu \mathrm{m}$ for the reacting flow. Firstly, the minimal and maximal sizes of droplets increase with the temperature. Besides, the increase of the minimal size is more important than the increase of the maximal size. Secondly, the coefficient of variation defined as the ratio of the standard deviation to the mean - is lower for reacting case than for the nonreacting case, which means that the dispersion on droplet size is less significant. Thirdly, the moment coefficient of skewness - or the 

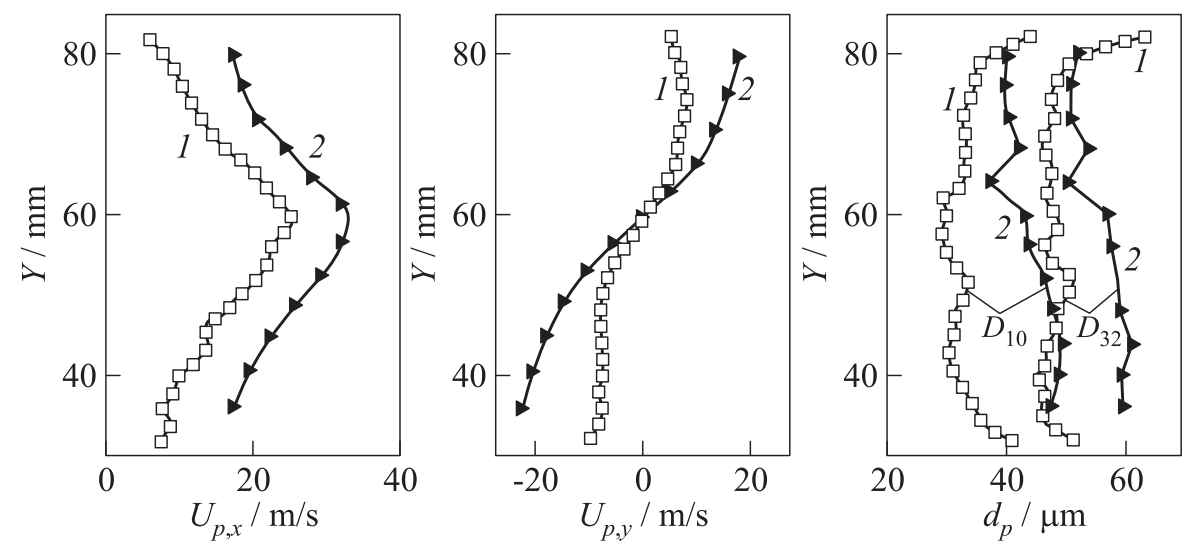

(a)
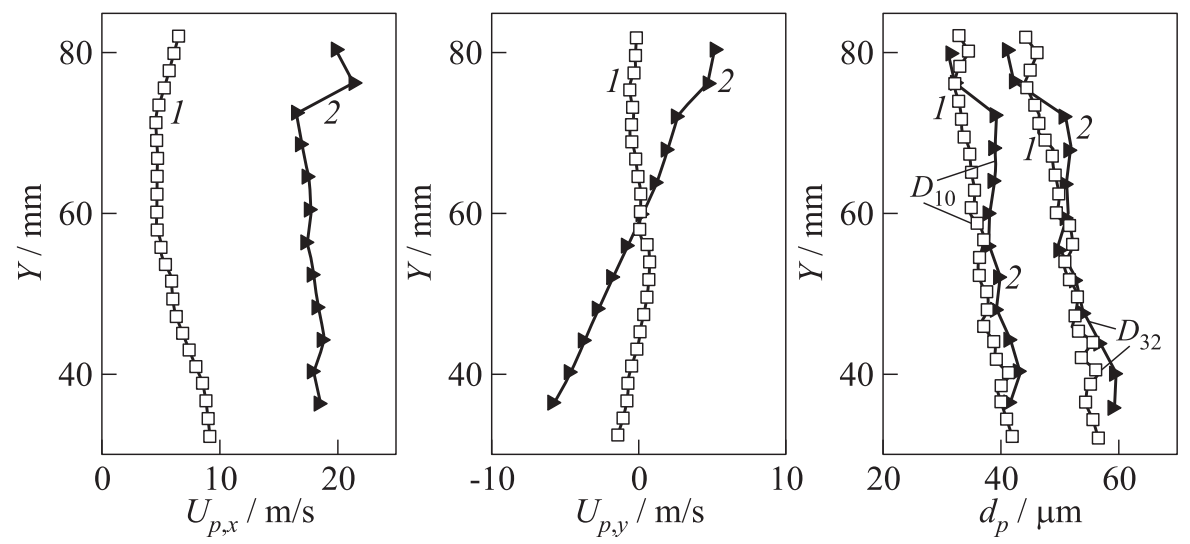

(b)

Figure 6 The PDI measurements sections at $9(a)$ and $53 \mathrm{~mm}(b): 1$ - nonreacting two-phase flow; and 2 - reacting two-phase flow

third standardized statistical moment - is smaller in the reacting flow; so, the size droplet distribution is straightened towards the biggest droplet sizes and becomes more symmetric around the arithmetic mean diameter. Lastly, the sample excess kurtosis - defined as the fourth standardized statistical moment - is also reduced under reactive conditions, which suggests that the droplet size distribution has a more rounded peak around the arithmetic mean diameter. Moreover, it implies thinner tails; thus, the distribution becomes narrower as surrounding gas temperature increases. Those tendencies can be explained by the fact that in the reacting flow, the smallest droplets are vanishing more rapidly than the 
biggest ones evaporate. This results in an increase of the mean diameters close to the fuel injector.

One observes from the arithmetic mean diameter profiles that the droplets which cross the flame (at $53 \mathrm{~mm}$ ) are smaller compared to the droplets located just upstream the flame (at $9 \mathrm{~mm}$ ). Moreover, the 53-millimeter section corresponds with the intersection of the $D_{10}$ profiles under nonreactive and reactive conditions. So, in the reacting flow, the droplets evaporate more rapidly due to their crossing in the flame. Further away in the flame, we expect to achieve even smaller droplets, until their complete disappearance further downstream.

At $53 \mathrm{~mm}$ from the nozzle, the average longitudinal velocity is flatter than at $9 \mathrm{~mm}$ (for the two conditions). Indeed, the flow is more uniform because it is less influenced by the bluff-body's wake. Moreover, the droplets are likely in equilibrium with the gaseous phase.

As one sees on PDI velocity profile, the droplets are accelerated in the reacting flow compared to the nonreacting flow. This is probably due to the gas expansion with combustion.

\subsection{Spatial Distribution of Droplets}

The droplet distribution is of fundamental importance in understanding of physical mechanisms taking place in combustor sprays. The aim of this section is to characterize the statistical spatial distribution of fuel droplets in the reacting flow. In order to do so, three analysis windows in the upper part of the combustion chamber have been studied. Each window is defined by its center point in millimetres: I $(17,52)_{X, Y}$, II $(31,63)_{X, Y}$, and III $(49,76)_{X, Y}$ and its size extension. The three points are represented in Fig. $5 b$. Moreover, Table 2 summarizes

Table 2 Outcomes of the experimental distributions of droplets - reacting two-phase flow

\begin{tabular}{|c|c|c|c|c|c|}
\hline Point & $\begin{array}{c}\text { Window size, } \\
\mu \mathrm{m} \\
\left.\text { (sample size }^{*}\right) \\
\end{array}$ & $\begin{array}{l}\text { Distribution } \\
\text { fit }\left[n^{* *}\right] \\
(\mathbb{E}[n], \mathrm{SD}[n]) \\
\end{array}$ & $\begin{array}{c}\text { Distribution } \\
\text { fit }\left[D_{i}\right] \\
\left(\mathbb{E}\left[D_{i}\right], \mathrm{SD}\left[D_{i}^{* * *}\right]\right) \\
\end{array}$ & $\begin{array}{c}D_{i}^{\mathrm{unif}}, \\
\mu \mathrm{m}\end{array}$ & $\bar{C}=\frac{\mathbb{E}\left[D_{i}\right]}{D_{10}}$ \\
\hline I & $\begin{array}{c}993 \times 993 \\
(38000)\end{array}$ & $\begin{array}{l}\text { Log-Normal } \\
(1929,393)\end{array}$ & $\begin{array}{c}\text { Log-Normal } \\
(178,61)\end{array}$ & 232 & 4 \\
\hline II & $\begin{array}{c}3319 \times 3319 \\
(56100)\end{array}$ & $\begin{array}{l}\text { Log-Normal } \\
(258,125)\end{array}$ & $\begin{array}{c}\text { Log-Normal } \\
(365,217)\end{array}$ & 677 & 9 \\
\hline III & $\begin{array}{c}5673 \\
(27900) \\
\end{array}$ & $\begin{array}{l}\text { Log-Normal } \\
(44,30)\end{array}$ & $\begin{array}{l}\text { Log-Normal } \\
(860,721)\end{array}$ & 1745 & 22 \\
\hline
\end{tabular}

${ }^{*}$ Sample size in number of droplets.

${ }^{* *} n$ in number of droplets $/ \mathrm{cm}^{2}$.

${ }^{* * *} D_{i}$ in $\mu \mathrm{m}$. 
the main results obtained in the three analysis windows. The second column displays the area of each window and the sample size associated (total number of droplets). The third and fourth columns show the distribution fit of the parameters $n$ (number density of droplets) and $D_{i}$ (center-to-center interdroplet distance with respect to the nearest neighbor), respectively. $\mathbb{E}[X]$ and $\mathrm{SD}[X]$ are, respectively, the expected value and the standard deviation of the variable $X$ (either $D_{i}$ or $\left.n\right)$. The fifth column introduces the $D_{i}^{\text {unif }}$ parameter which designates the average interdroplet distance if all droplets were uniformly distributed in the windows (i.e., arrayed with regular intervals, equidistant droplets). The last column shows the corresponding distance parameter at each point.

To provide those results, a stack of Mie scattering images acquired at $1 \mathrm{kHz}$ is used. Each image is indexed with the superscript " $R$ " (for Repeated measurement). The stack height of images $L^{R}$ is equal to 2000 images. All the images are firstly processed by a tracking particle algorithm which provides the number of droplets by window $N^{R}$ for each image " $R$." Since the droplet concentration in each window is different, the windows are sized to have a sufficient size sample as

$$
N=\sum_{R=1}^{L^{R}} N^{R}>10000 .
$$

A window dimension influence study is performed by considering windows twice as large or twice as small and shows that the final results are not dependent of the window size. One defines the number density of droplets $n^{R}$ as the ratio of the number of droplets $N^{R}$ to the surface area of the window studied. Each counted droplet by window is indexed with the superscript " $S$ " (for Sample).

A second algorithm assesses the Euclidean distance between all the droplets identified in the window. More especially, $D_{i}^{R, S}$ is defined as the center-to-center distance between the droplet " $S$ " and its nearest neighbor in the window of image " $R$." In practice, this interdroplet distance allows to describe the level of dropletdroplet interactions in a spray (alteration of droplet drag and vaporization rates). Thus, $\mathbb{E}\left[D_{i}\right]$ are defined as the average of the nearest neighbor distances for all images and all droplets counted in the window:

$$
\mathbb{E}\left[D_{i}\right]=\frac{1}{N} \sum_{R=1}^{L^{R}} \sum_{S=1}^{N^{R}} D_{i}^{R, S} .
$$

In order to compare the average interdroplet distance measured experimentally $\mathbb{E}\left[D_{i}\right]$ to a reference droplet spacing, the $D_{i}^{\text {unif }}$ parameter is defined as the inter-droplet distance if all droplets were uniformly distributed in the windows. This theoretical parameter can be written as

$$
D_{i}^{\text {unif }}=\frac{1}{L^{R}} \sum_{R=1}^{L^{R}}\left[n^{R}\right]^{-1 / 2} .
$$




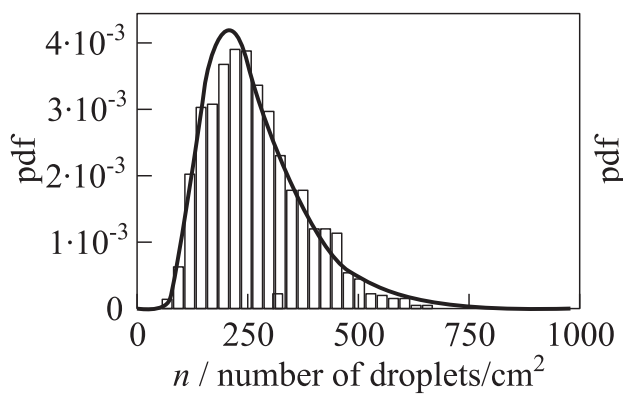

(a)

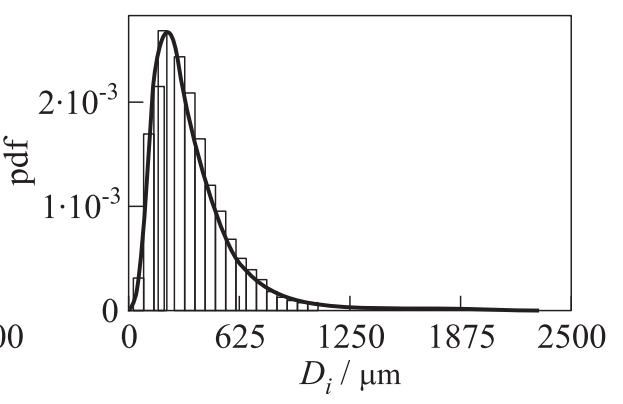

(b)

Figure 7 Probability density functions of the number $(a)$ and the interdroplet distance $(b)$ for reacting two-phase flow, point II (Log-Normal, $\mathbb{E}[n]=258$ number of droplets $/ \mathrm{cm}^{2} ; \mathrm{SD}[n]=125$ number of droplets $/ \mathrm{cm}^{2} ; \mathbb{E}\left[D_{i}\right]=365 \mu \mathrm{m} ;$ and $\left.\mathrm{SD}\left[D_{i}\right]=217 \mu \mathrm{m}\right)$

Firstly, from Table 2, one notes that the uniform (or regular) distance is always greater than the average interdroplet distance measured experimentally $\left(D_{i}^{\text {unif }}>\mathbb{E}\left[D_{i}\right]\right)$. To go further into this observation, fit distributions are carried out from the statistics in number density and interdroplet distance. Among a vast choice of pdf's, the best distribution fitting is searched (i. e., which minimizes the value of the Bayesian information criterion). The outcomes are displayed in Table 2. It appears that all the interdroplet distance distributions follow positively skewed distributions, in particular, Log-Normal. Therefore, those distributions are shifted towards the smallest value of interdroplet distance. Likewise, the number density of droplets may be approximated with Log-Normal pdf's. In Fig. 7, distributions are fitted from the experimental histograms for the point II and one can see the goodness-of-fit. In conclusion, these results suggest that the assumption of uniform (or regular) distribution of droplets made in analytical studies of spray combustion regimes can be refuted $[1,3,5]$.

Secondly, from Table 2, another observation is that the number density decreases with the distance from the nozzle and, conversely, the interdroplet distance increases. One assumes that there must be a dependence between those parameters. Consequently, we attempt to relate $\overline{D_{i}^{R}}$, defined as the average of the nearest neighbor distances for the image " $R$ " (see Eq. (3)), and $n^{R}$, the number density of droplets for the image " $R$." By doing so, one can formulate the dependence between those two parameters (see Eq. (4)):

$$
\begin{aligned}
\overline{D_{i}^{R}} & =\frac{1}{N^{R}} \sum_{S=1}^{N^{R}} D_{i}^{R, S} \\
& =\alpha^{R}\left[n^{R}\right]^{-1 / 2}
\end{aligned}
$$


where $\alpha^{R}$ is the dimensionless proportional coefficient between 0 and 1. For instance, if the coefficient $\alpha^{R}$ is equal to unity, one finds the theoretical uniform distribution.

In order to conclude about the droplet clustering, the spatial distribution of droplets measured experimentally has been compared with a random distribution. For this purpose, a program including a pseudorandom number generator is implemented for generating random droplet positions in each window whose dimensions are equal to the experimental ones. A lot of simulations, more than 5000 , are conducted to produce statistics on interdroplet distances. Subsequently, by defining distinct classes of number density, a subroutine sorts the data and calculates an average interdroplet distance per class $\mathbb{E}\left[D_{i} \mid n\right]$. In particular, $\mathbb{E}\left[D_{i} \mid n\right]$ is defined as the conditional expected value of interdroplet distance knowing $n$ and, hence, can be expressed by Eq. (5). Besides, in the subroutine, the number density of droplets inside each window varies from minimal to maximal values which are close to the measured ones. In addition, this interval is arbitrarily cut in 6 classes. Finally, the subroutine assesses the coefficient $\alpha_{\mid n}$ from Eq. (6) in order to characterize the distribution:

$$
\begin{aligned}
\mathbb{E}\left[D_{i} \mid n\right] & =\frac{1}{N_{\mid n}} \sum_{R=1}^{L^{R} \mid n} \sum_{S=1}^{N^{R} \mid n} D_{i}^{R, S} \\
& =\alpha_{\mid n} n^{-1 / 2}
\end{aligned}
$$

where $N_{\mid n}, N_{\mid n}^{R}$, and $L_{\mid n}^{R}$ are the total number of droplets, the number of droplets by window for the image " $R$," and the stack height of images, respectively, and are all conditioned for a fixed $n$.

To compare those numerical calculations with the experiments, the same subroutine is used to process the experimental data. The variation of the average interdroplet distance per class vs. the inverse square root of the number density, at the point II, for the uniform, experimental, and random distributions is plotted in Fig. 8.

At first, it seems that for each distribution, the coefficient $\alpha_{\mid n}$ is constant. In order words, the proportional coefficient between $\mathbb{E}\left[D_{i} \mid n\right]$ and $n$ is independent of the number of droplets in the window. So, from Table 2, one can obtain a good assessment of the experimental value of $\alpha_{\mid n}$ by solely determining the ratio of $\mathbb{E}\left[D_{i}\right]$ and $D_{i}^{\text {unif }}$. From Fig. 8, it appears no significant deviation of the spatial distribution of droplets between experimental and a perfectly random distribution. Equivalent outcome is found for the other analysis points $[12,17]$. In consequence, under those reactive conditions, there are no more neighbor droplets than random case and, thus, no evidence of droplet clustering is observed within the flame. Therefore, droplets seem mainly scattered by random phenomena, such as turbulence, droplet collisions but not much influenced by additional causes - such as memory of the injection process — which may lead 


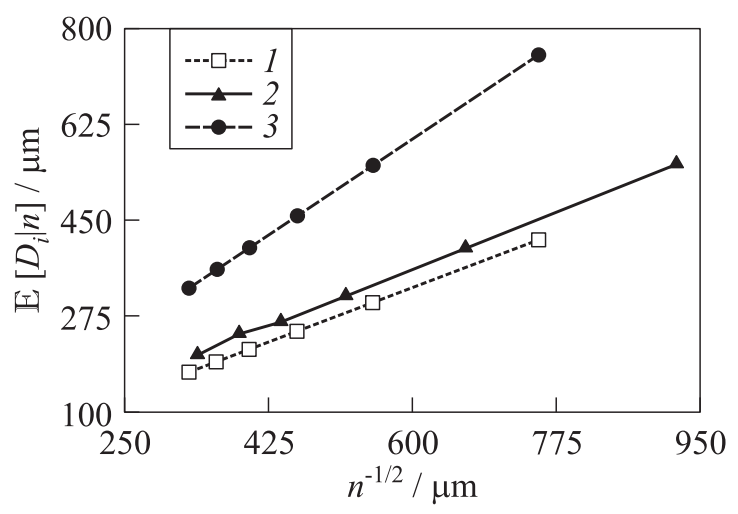

Figure 8 Variation of the conditional average interdroplet distance vs. the inverse square root of the density number for different types of distributions: 1 - random, $\alpha_{\mid n}=0.53 ; 2-$ experimental, $\alpha_{\mid n}=0.59 ;$ and $3-$ uniform, $\alpha_{\mid n}=1$. Reacting two-phase flow, point II

to droplet clustering formation (or "patchiness") as suggested by Kostinski and Shaw [18].

In this study, the laser sheet is about 1-millimeter thickness. More advanced analysis made in $[12,17]$ showed that the nonzero value of thickness generates two kinds of errors during the processing, namely, a projection error and a restriction error (or border effect). In the investigated case, we numerically estimate that the total error on the average of the nearest neighbor distances is about $\pm 20 \%$. Possible improvements would be to reduce the laser sheet thickness or to extend the analysis window sizes. The former is difficult to reach without reducing the laser sheet intensity. The latter leads to a trade-off between two constraints, namely, a sufficient number of samples (droplets) and the local aspect of the measurement.

\subsection{Spray Combustion Regimes}

In the introduction, a selection of experimental studies dealing with spray combustion physics has been presented. It appears that a measurement device which allows to simultaneously visualize droplets and reaction zones offers interesting prospects to describe spray flame structure and to highlight the existence of spray combustion regimes. For this study, two optical planar diagnostic techniques, namely, tomography laser (Mie scattering) and PLIF imaging on hydroxyl radicals $(\mathrm{OH})$ are synchronized in order to benefit from the bidimensional property of the experimental setup. A description of this measurement device was presented in subsection 2.3. Two visualization windows have been defined and correspond, 

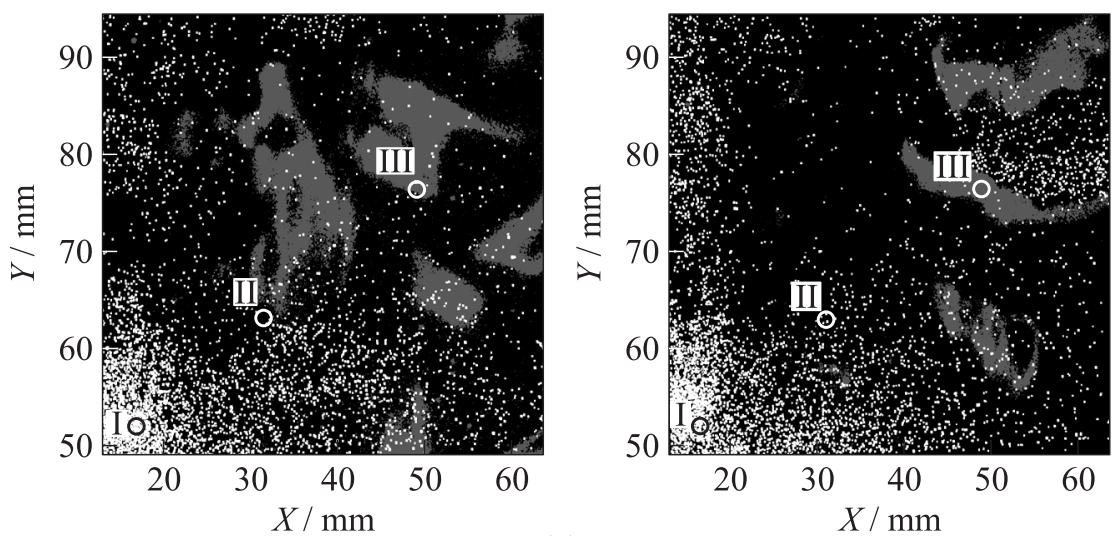

(a)
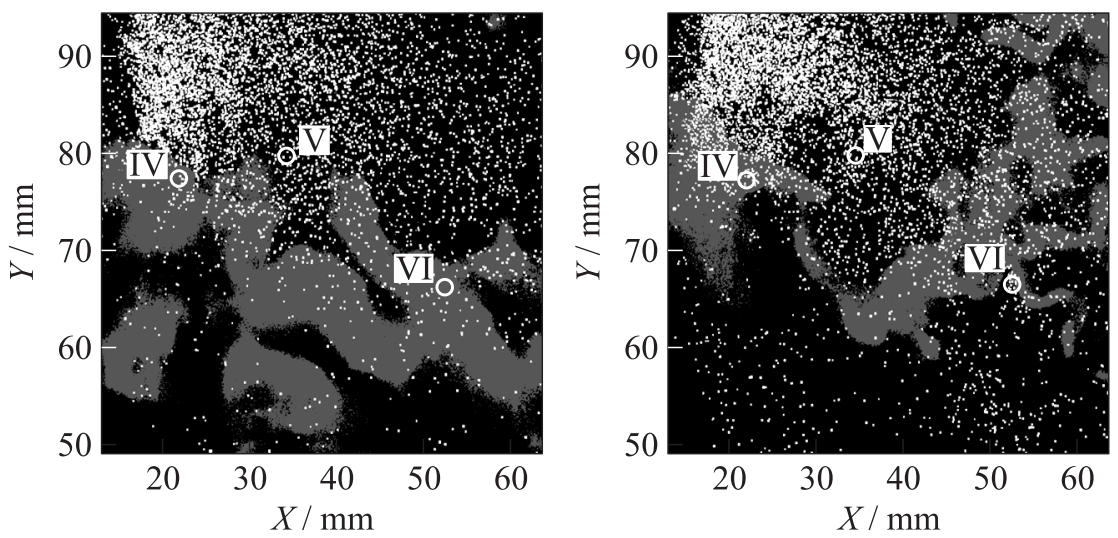

(b)

Figure 9 Four instantaneous images of simultaneous OH-PLIF (left column) and Mie scattering (right column), reacting two-phase flow: $(a)$ upper part of the combustion chamber; and $(b)$ lower part of the combustion chamber

respectively, to the top and to the bottom of the combustion chamber. Figures $9 a$ and $9 b$ show different instantaneous cross-section views of the spray flame. These images show both the droplet positions (in white) and the hot gases/reacting zones (in grey). Note that the locations of the analysis windows are also displayed.

First, one observes that the number of droplets is relatively important close to the fuel injector and it significantly decreases through the flame. Such an evolution is consistent with the presence of the flame involving high temperature and rapid evaporation of droplets. One notes also that the flow is characterized 
by strong heterogeneities in spatial distribution of droplets and reaction zones. This behavior is different from a purely gaseous combustion in which the flame is much more homogeneous.

In the upper part of the combustion chamber (see Fig. 9a), the flame appears fragmented into "reactive pockets" (or pockets of burnt gases). The presence of nonreactive zones in the spray can be explained by local conditions which are outside the flammability limits. Nevertheless, it is difficult to confirm this assumption since none information about local fuel concentration is available here. Finally, one notes that this discontinuous flame behavior organized in "reactive pockets" (or pockets of burnt gases) seems similar to the "pocket combustion" regime proposed by Borghi and Champion [19].

In the lower part of the combustor (see Fig. $9 b$ ), the visualizations show a higher density droplets than in the upper part. One observes also that the reaction zones are much more grouped together (i.e., flame less fragmented). Moreover, the flame is established outside the fuel feeding zone (i.e., at the periphery of the dense area of droplets) probably because of a too rich mixture. As a consequence, it seems that an "external group combustion" regime occurs in this part of the flow, according to the classification of Chiu and Kim [1].

As reported in Table 2 , the distance parameter $\bar{C}=\mathbb{E}\left[D_{i}\right] / D_{10}$ is equal to 4 at the point I. Since the number density is high, the collective droplet effects must be important and the blowing rate of fuel vapor may preclude the oxygen penetration close to the injection system. The distance parameter increases with the distance to the fuel injector. At the point III, for instance, the distance parameter is about 22 and it corresponds to a diluted region of flow, where the heat released by the combustion process is important (see Fig. $5 b$ ). According to Crowe et al. [20], when this distance parameter is above 10, one can consider that droplets are sufficiently distant from each other to behave as isolated droplets (without interaction). In this study, the resolution of PLIF-OH/Mie images may be insufficient to highlight a "single droplet combustion" regime (i.e., diffusion flame enclosing individual droplet).

In conclusion, it seems clear that the interdroplet distance (nearest neighbor) plays a major role in the appearance of these different spray combustion regimes. In perspective, knowledge of the local equivalence ratio, which requires to measure the local mass fraction of fuel and air, would particularly useful for characterizing spray combustion regimes in more details.

\section{CONCLUDING REMARKS}

The challenge of this experimental work was to build a new database in a twophase flow combustion (nonpremixed spray) in order to improve spray combustion modeling in numerical codes. For this purpose, at ONERA Fauga- 
Mauzac, a new air-breathing propulsion test setup has been recently developed: Prométhée-LACOM. The configuration is rather academic but some features of the flow in an actual aeroengine combustor are preserved (like the spray polydispersity or the equivalence ratio). Moreover, because of its bidimensional design, the test setup is particularly suitable for optical diagnostics. In this paper, both nonreacting and reacting two-phase flows were investigated.

To characterize the nonreacting flow, measurements on the gaseous phase (pressure fluctuations) and the liquid phase (two-components PDI and highspeed Mie scattering by droplets imaging) were carried out. A spectral analysis from the pressure fluctuations just upstream the combustor revealed a narrowband peak frequency corresponding with a von Kármán vortex shedding. A phase-averaging was applied on the Mie scattering images and permitted to relate the unsteadiness of droplets dispersion with regards to the quasi-periodic dynamic of the gaseous phase. Two PDI measurements sections were provided in order to define the injection conditions for numerical simulations (droplet sizes and velocities).

Likewise, a spectral analysis from the pressure fluctuations was conducted under reactive conditions. Nevertheless, the complete vanishing of the vortex shedding because of heat released by the combustion process was observed. The same two PDI measurement sections were realized and compared with the nonreactive case. Firstly, differences on droplet size distributions were noticed. In the reacting flow, the smallest droplets were vanishing more rapidly than the biggest ones evaporate. Through the flame, the droplet size decreased rapidly. Secondly, the droplets were accelerated in the reacting flow compared to the nonreacting flow.

Another important point of this study was to characterize the spatial distribution of fuel droplets in the reacting flow and to perform statistical analysis from a large stack of Mie scattering images. One key finding is that the centerto-center interdroplet distance (nearest neighbor) can be described by means of a perfectly random distribution. Therefore, it can be concluded that the assumption of uniform (or regular) distribution of droplets made in analytical studies of spray combustion regimes can be refuted. Furthermore, the dependency between the interdroplet distance and the inverse square root of the number density was generalized by introducing a proportional coefficient (see Eq. (6)). Moreover, no evidence of droplet clustering was observed within the flame.

At last, simultaneous OH-PLIF and Mie scattering imaging were implemented in order to describe the spray flame structure. By using such superimposed images, detailed cross sections of the two-phase flow combustion were obtained. The different behaviors observed in this study seem to support the existence of spray combustion regimes. For instance, near the injector, the flame is established outside the fuel feeding zone because of a too rich mixture. Within a less dense zone, it seems that a "pocket combustion" regime was observed. Further away, the interdroplet distances become so important that droplets can 
be considered as isolated. In conclusion, it seems clear that the spacing between droplets is one of the most important parameter to describe collective droplet effects (alteration of drag and vaporization rate) and the spray combustion regimes.

\section{ACKNOWLEDGMENTS}

The authors greatly acknowledge the financial support by the Midi-Pyrénées Region and the European Community.

\section{REFERENCES}

1. Chiu, H. H., and H. Y. Kim. 1983. Group combustion of liquid fuel sprays. AIAA Paper No. 83-150.

2. Chigier, N.A. 1983. Group combustion models and laser diagnostic methods in sprays: A review. Combust. Flame 51:127-139.

3. Correa, S. M., and M. Sichel. 1982. The group combustion of a spherical cloud of monodisperse fuel droplets. Proc. Combust. Inst. 19:981-991.

4. Annamalai, K., and W. Ryan. 1992. Interactive processes in gasification and combustion. Part I: Liquid drop arrays and clouds. Prog. Energ. Combust. 18:221-295.

5. Candel, S., N. Lacas, N. Darabiha, and C. Rolon. 1999. Group combustion in spray flames. Multiphase Sci. Technol. 11:1-18.

6. Reveillon, J., and L. Vervisch. 2005. Analysis of weakly turbulent dilute-spray flames and spray combustion regimes. J. Fluid. Mech. 537:317-347.

7. Akamatsu, F., Y. Mizutani, M. Katsuki, S. Tsushima, and Y. Dae Cho. 1996. Measurement of the local group combustion number of droplet clusters in a premixed spray stream. Proc. Combust. Inst. 26:1723-1729.

8. Akamatsu, F., S. M. Hwang, and H.-S. Park. 2007. Evaluation of combustion mechanism of droplet cluster by simultaneous time-series measurements in premixed spray flame. J. Ind. Eng. Chem. 13:206-213.

9. Beck, C. H., R. Koch, and H.-J. Bauer. 2008. Investigation of the effect of incomplete droplet prevaporization on NOx emissions in LDI combustion systems. J. Eng. Gas Turb. Power 130:1-8.

10. Beck, C. H, R. Koch, and H.-J. Bauer. 2009. Identification of droplet burning modes in lean, partially prevaporized swirl-stabilized spray flames. Proc. Combust. Inst. 32:2195-2203.

11. Vicentini, M., R. Lecourt, V. Bodoc, O. Rouzaud, and O. Simonin. 2014. Régimes de combustion dans les foyers aéronautiques: Mise en place expérimentale de l'écoulement diphasique. Congrés Francophone des Techniques Laser 14:LV-5.1. 
12. Vicentini, M. 2016. Mise en évidence expérimentale et modélisation des régimes de combustion diphasique présents dans les foyers aéronautiques. University Toulouse. Ph.D. Thesis.

13. Bachalo, W. D. 2000. Spray diagnostics for the twenty-first century. Atomization Spray. 10:439-474.

14. Bill, R. G., and K. Tarabanis. 1986. The effect of premixed combustion on the recirculation zone of circular cylinders. Combust. Sci. Technol. 39-53.

15. Shanbhogue, S. J., S. Hussain, and T. Lieuwen. 2009. Lean blowoff of bluff-body stabilized flames: Scaling and dynamics. Prog. Energ. Combust. Sci. 35:98-120.

16. Chaudhuri, S., S. Kostka, M. W. Renfro, and B. M. Cetegen. 2010. Blowoff dynamics of bluff-body stabilized turbulent premixed flames. Combust. Flame 157:790802.

17. Rouzaud, O., M. Vicentini, R. Lecourt, V. Bodoc, and O. Simonin. 2016. Experimental analysis of droplet spatial distribution in a spray burner. ILASS Proceedings. $10 \mathrm{p}$.

18. Kostinski, A. B., and R. A. Shaw. 2001. Scale-dependent droplet clustering in turbulent clouds. J. Fluid. Mech. 434:389-398.

19. Borghi, R., and M. Champion. 2000. Modélisation et théorie des flammes. Editions Technip. $384 \mathrm{p}$.

20. Crowe, C., J. D. Schwarzkopf, M. Sommerfeld, and Y. Tsuji. 2011. Multiphase flows with droplets and particles. CRC Press Book. 509 p. 